\title{
Effective and efficient use of implantable defibrillators: Sometimes it's over when it's over
}

\author{
Paul Dorian MD
}

$\infty \quad$ See related research paper by Setoguchi and colleagues, page 611

$\mathrm{P}$ atients with substantial left ventricular scarring are prone to sudden death, presumably from ventricular tachyarrhythmias (sustained ventricular tachycardia and ventricular fibrillation). Rates of death, especially sudden cardiac death, increase as left ventricular function worsens. At first glance, implantable cardioverter defibrillators seem to offer a simple solution to this complex problem. It appears self-evident that the implantation of a device that has a greater than $98 \%$ probability of successfully terminating lethal ventricular arrhythmias would be life-prolonging in most if not all such patients. Indeed, randomized clinical trials of defibrillators implanted in patients at risk of sudden death because of severe left ventricular dysfunction (usually defined as an ejection fraction $<30 \%$ ) have shown a $20 \%-30 \%$ decrease in allcause mortality and a $50 \%-75 \%$ decrease in the rate of sudden death. ${ }^{1}$ Hence, on the premise that "it ain't over 'til it's over," defibrillators are often implanted prophylactically in patients with severe left ventricular dysfunction.

However, not all patients benefit equally from implantable defibrillators. The devices cannot effectively prevent deaths from causes other than arrhythmia. Also, the number of patients needed to treat to reduce all-cause mortality by a meaningful amount is directly related to the risk of death from arrhythmia, as opposed to the risk of overall death. ${ }^{2}$ Thus, to ensure that the use of implantable defibrillators is costeffective and efficient (i.e., to avoid the morbidity and complications of defibrillator placement in patients destined never to receive therapy from the device), it is important to understand the clinical characteristics of patients who will be most (or least) likely to benefit from an implantable defibrillator.

In this issue of CMAJ, Setoguchi and colleagues provide an example, among others previously observed, of a patient population in which implantable defibrillators are unlikely to provide a meaningful survival benefit. ${ }^{3}$ In their cohort of a largely elderly population with at least 1 previous hospital admission related to heart failure, they found that $13.7 \%$ of the deaths occurred outside a hospital setting. This finding suggests that only a small proportion of deaths are likely to be sudden and due to arrhythmia. In their appropriately conservative analysis, the authors assumed that all out-of-hospital deaths were potentially preventable with the use of an implantable defibrillator, which is most unlikely.

However, the authors' expression of the measure of potential benefit from implantable defibrillators as the prolongation of a hypothetical median survival is not clinically useful. The absolute survival benefit will be realized in only a small pro-

\section{Key points}

- Although implantable defibrillators are highly effective in preventing sudden cardiac death, not all patients benefit from them.

- The study by Setoguchi and colleagues suggests that patients with heart failure who have prior hospital admissions related to heart failure and those who have chronic kidney disease are at too high a risk of death from non-arrhythmia causes to benefit from the device.

- Use of implantable defibrillators will likely also not benefit patients with heart failure at low risk of death, such as those with an ejection fraction of more than $30 \%$ and a short QRS interval.

- Clinical decisions regarding defibrillator implantation should consider effective use of resources and whether a reasonable balance exists between benefit and risk.

portion of patients, who as individuals are likely to derive substantial benefit if they survive long enough after a successful defibrillator shock. Other limitations of the study include uncertainty about cause of death, and thus its preventability, the implicit assumption that no in-hospital deaths would be preventable with the use of an implantable defibrillator, the absence of data on left ventricular function, and the collection of data at a time when currently indicated drug therapies such as $\beta$-blockers, angiotensin-converting-enzyme inhibitors or angiotensin-receptor blockers, and statins (in patients with coronary artery disease) were used less often.

Nevertheless, the study by Setoguchi and colleagues reminds us that, in some patient populations, the decision to implant a defibrillator for primary prophylaxis should be made very carefully. For some patients with left ventricular dysfunction, defibrillators simply do not prolong survival, as evidenced by the results of large multicentre randomized controlled trials. ${ }^{4}$ In the Defibrillator in Acute Myocardial Infarction Trial (DINAMIT), patients were randomly assigned to receive an implantable defibrillator or no defibrillator shortly after an acute myocardial infarction associated with an ejection fraction of less than $35 \%$ and decreased heart rate variability (an indicator of increased risk of death). ${ }^{5.6} \mathrm{~Pa}-$ tients who received a defibrillator did not live longer than those given standard pharmacotherapy alone. The benefit of the defibrillators in reducing sudden cardiac death was offset

Paul Dorian is Professor and Director, Division of Cardiology, University of Toronto, Toronto, Ont. 


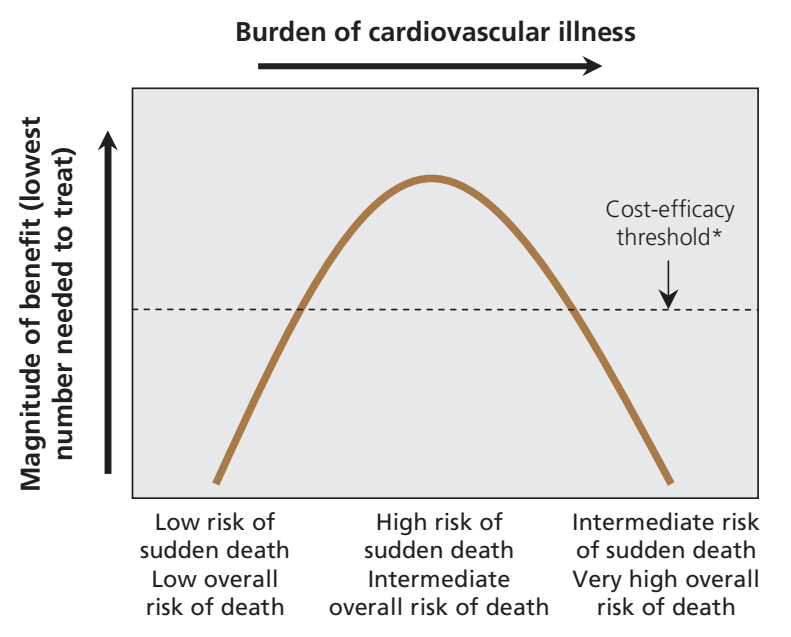

Figure 1: U-shaped relation between number needed to treat with an implantable defibrillator and overall burden of cardiovascular disease. *The cost-efficacy threshold can be arbitrarily set to any value, depending on resources and consensus.

by an increased risk of other causes of death, presumably from myocardial ischemic events or heart failure.

In the Coronary Artery Bypass Graft (CABG) Patch Trial, patients with an ejection fraction of less than $35 \%$ and a positive signal-averaged electrocardiogram (another marker of poor outcome) were randomly assigned to receive an implantable defibrillator or no defibrillator immediately after bypass surgery. ${ }^{7,8}$ Defibrillators did not prolong survival. Despite a substantial proportion of patients receiving "appropriate defibrillator therapy," the presumed reduction in sudden death was offset by an increase in other kinds of cardiovascular-related death.

These randomized controlled trials lend credence to the "conversion hypothesis," which suggests that, in some patient populations, a successful defibrillator shock will prevent sudden cardiac death but that the "saved" patient will be at high risk of dying from another cardiovascular cause within a short period. The benefit of the defibrillator is thus negated.

In other patient populations, use of an implantable defibrillator may be relatively ineffective because the underlying risk of sudden death is very low, or because the risk of death from other causes is so high that the probability of a meaningful prolongation of life for the majority of patients is small. Although information is sparse, patients with an ejection fraction of more than $30 \%$, particularly those with a QRS interval of less than $120 \mathrm{~ms}$, appear to be at low risk of cardiovascular-related death; therefore, these patients will likely not experience a major longterm benefit from implantable defibrillators. ${ }^{9} 10$ At the other end of the spectrum, as Setoguchi and colleagues point out, patients with heart failure at high risk of death, including those with prior (especially multiple) hospital admissions related to heart failure and those with chronic kidney disease, are probably at too high a risk of death from non-arrhythmia causes to benefit from defibrillator implantation. Even if all of the $13.7 \%$ of potentially treatable sudden deaths were prevented with an implantable defibrillator, the overall risk of death would remain prohibitively high. These hypotheses are supported by the seemingly counterintuitive observation in the largest trial of prophy- lactic use of implantable defibrillators, the Sudden Cardiac Death in Heart Failure Trial (SCD-HeFT): patients with more severe heart failure (New York Heart Association class III) received less benefit from an implantable defibrillator than patients with class II symptoms of heart failure. ${ }^{11}$

These concepts lead one to believe that a "one size fits all" approach to the use of implantable defibrillators may represent neither the most effective use of resources nor the best balance between risk and benefit. There is likely a U-shaped relation between the number needed to treat with an implantable defibrillator to prolong life and a patient's overall burden of cardiovascular illness (Figure 1). Some patients have a sufficiently low risk of sudden death that defibrillators may not be warranted. As the burden of illness increases, use of implantable defibrillators may become increasingly effective in terms of an absolute reduction of mortality, until morbidity and the risk of death from causes other than sudden death become sufficiently high to make defibrillators again less useful.

As in all areas in medicine, appropriate decision-making regarding the use of implantable defibrillators requires thoughtful consideration of both the clinical trial evidence and the clinical context.

Competing interests: Paul Dorian has received speaker fees and educational grants from Boston Scientific Inc., Medtronic Inc. and St. Jude Inc.

Acknowledgement: I am grateful to Dr. Arnold Pinter for his helpful comments and suggestions.

\section{REFERENCES}

1. Nanthakumar K, Epstein AE, Kay GN, et al. Prophylactic implantable cardioverterdefibrillator therapy in patients with left ventricular systolic dysfunction: a pooled analysis of 10 primary prevention trials. J Am Coll Cardiol 2004;44:2166-72.

2. Jolly S, Dorian P, Alter DA. The impact of implantable cardiac defibrillators for primary prophylaxis in the community: baseline risk and clinically meaningful benefits. J Eval Clin Pract 2006;12:190-5.

3. Setoguchi S, Nohria A, Rassen JA, et al. Maximum potential benefit of implantable defibrillators in preventing sudden death after hospital admission because of heart failure. CMAJ 2009;180:611-6.

4. Myerburg RJ. Implantable cardioverter-defibrillators after myocardial infarction. $N$ Engl J Med 2008;359:2245-53.

5. Hohnloser SH, Kuck KH, Dorian P, et al.; DINAMIT Investigators. Prophylactic use of an implantable cardioverter-defibrillator after acute myocardial infarction. N Engl J Med 2004;351:2481-8.

6. Dorian P, Connolly S, Hohnloser SH; DINAMIT Investigators. Why don't ICD's decrease all-cause mortality after MI? Insights from the DINAMIT Study [abstract]. Circulation 2004;110:III-502.

7. Bigger JT Jr; Coronary Artery Bypass Graft (CABG) Patch Trial Investigators. Prophylactic use of implanted cardiac defibrillators in patients at high risk for ventricular arrhythmias after coronary-artery bypass graft surgery. N Engl J Med 1997; 337:1569-75.

8. Bigger JT Jr, Whang W, Rottman JN, et al. Mechanisms of death in the CABG Patch Trial: a randomized trial of implantable cardiac defibrillator prophylaxis in patients at high risk of death after coronary artery bypass graft surgery. Circulation 1999;99:1416-21.

9. Tang AS, Ross H, Simpson CS, et al.; Canadian Heart Rhythm Society, Canadian Cardiovascular Society. Canadian Cardiovascular Society/Canadian Heart Rhythm Society position paper on implantable cardioverter defibrillator use in Canada. Can J Cardiol 2005;21(Suppl A):11A-8A.

10. Dorian P, Talajic M, Levesque S, et al.; AF-CHF Investigators. Prolonged QRS duration is associated with a marked increase in mortality in the AF-CHF Study. Circulation 2008;118:S795.

11. Bardy GH, Lee KL, Mark DB, et al.; Sudden Cardiac Death in Heart Failure Trial. (SCD-HeFT) Investigators. Amiodarone or an implantable cardioverter-defibrillator for congestive heart failure. $N$ Engl J Med 2005;352:225-37.

Correspondence to: Dr. Paul Dorian, Division of Cardiology, St. Michael's Hospital, University of Toronto, 30 Bond St., Toronto ON M5B 1W8; fax 416 864-5104; dorianp@smh.toronto.on.ca 